\title{
Low Noise C-Band EDFA/DRA Hybrid Amplifier Using the Same Pump Laser Diodes
}

\author{
M.I. Azawe* \\ Department of Physics, College of Education, Mosul University, Mosul, IRAQ.
}

Received October 07, 2011; accepted November 07, 2011; published December xx, 2011

\begin{abstract}
This paper presents the investigation of a hybrid amplifier consisting of Erbium doped fiber amplifiers EDFAs and a distributed Raman amplifier in the C-band. Our simulations take into account all the effects associated with transmitted signals and a pump laser in the optical fiber. The overall noise figure for the proposed hybrid amplifier was $<10 \mathrm{~dB}$, as a limiting case for maximizing the span length and/or minimizing the impairments of fiber nonlinearities. The combination distributed Raman amplifier and EDFAs present better performance than conventional EDFA-only systems. This low noise figure will enable maximum transmission distance. The transmission is discussed of 16 channels spaced $1 \mathrm{~nm}$ and $1 \mathrm{~mW}$ of optical power through $100 \mathrm{~km}$ of fiber.
\end{abstract}

As the demand for higher transmission capacity in wavelength division multiplexing WDM systems increases, the channel speed, channel number, and spectral efficiency need to be upgraded [1]. To this end, Raman amplifiers have become essential in overcoming the limitations of the bandwidth, noise figure NF, and output power of conventional doped fiber amplifiers [2][8]. Recently, great attention has been paid to the Raman amplifiers used in conjunction with Erbium-doped fiber amplifiers EDFAs to form hybrid amplifiers, especially when the system capacity needs to be upgraded by raising channel speed and spectral efficiency without bandwidth expansion [3]-[8].

Hybrid EDFA/Raman amplifiers are also attractive in optical communications because of their abilities of tailoring gain profile, compensating fiber dispersion and loss, enhancing the optical signal-to-noise ratio [9]. These amplifiers are designed in order to maximize the span length and/or to minimize the impairments of fiber nonlinearities, and to enhance the bandwidth of EDFAs [10]. Recently, hybrid amplifiers known as Ramanassisted fiber optical parametric amplifiers RA-FOPAs have attracted much attention because of their flexibility in selecting parametric pump wavelengths and full utilization of the $\mathrm{C}+\mathrm{L}$ band in addition to the inherent advantages of FOPAs [11]. The gain profile in WDM systems must be carefully equalized during propagation or after short distances, which typically requires the use of additional gain flattening filters or multiple wavelengths

\footnotetext{
*E-mail: muzahim_935@yahoo.com
}

Raman pumps, adding to the cost of a fiber optic link. However, for applications in which a smaller channel bandwidth is sufficient, the gain equalization can be done using a simplified arrangement of an EDFA and singlepump distributed Raman amplification [12], as will be done in this investigation.

This paper describes the design of a narrowband hybrid amplifier composed of a distributed Raman amplifier and Erbium doped fiber amplifiers for the Cband, using the same pump lasers to estimate the noise figure of configuration. We characterize the design by numerical simulation based on the Optisystem 7.0 of an ordinary single mode fiber transmission line.

The gain (in $\mathrm{dB}$ ) of an EDFA at a signal wavelength $\lambda_{s}$ can be expressed as [13]:

$$
\begin{aligned}
G_{d B}\left(\lambda_{s}\right) & =\frac{10}{\ln (10)} \eta \rho L . \\
& \left\{\left(\sigma_{a b s}\left(\lambda_{s}\right)+\sigma_{e m i}\left(\lambda_{s}\right)-\sigma_{E S A}\left(\lambda_{s}\right)\right) N_{2 a v e}-\sigma_{a b s}\left(\lambda_{s}\right)\right\}
\end{aligned}
$$

with $N_{\text {2ave }}=\frac{1}{L} \int_{0}^{L} N_{2}(z) d z$

(2)

where $\eta$ is the overlap factor, $\rho$ is the Er concentration, $L$ is the EDF length, $\sigma_{a b s}, \sigma_{e m i}, \sigma_{E S A}$ are the absorption, emission and excited-state absorption ESA cross-section, respectively, and $N_{2 a v e}$ is the path-averaged fractional population of the upper state $\left(0<N_{2 a v e}<1\right) . N_{2}(z)$ is the population of the upper state as fiber length dependent. $G_{\mathrm{dB}}\left(\lambda_{\mathrm{s}}\right)$ is proportional to the concentration-length product $\rho L$, and the gain spectrum depends on the $N_{2 \text { ave }}$

The noise figure $N F$ of the EDFA, as a function of the signal wavelength $\lambda_{\mathrm{s}}$, can be expressed as [13]:

$$
N F\left(\lambda_{s}\right)=\frac{2 R\left(\lambda_{s}\right)}{R\left(\lambda_{s}\right)-R\left(\lambda_{p}\right)-\sigma_{E S A}\left(\lambda_{s}\right) / \sigma_{a b s}\left(\lambda_{s}\right)}
$$

with

$$
R(\lambda) \equiv \frac{\sigma_{e m i}(\lambda)}{\sigma_{a b s}(\lambda)}
$$

where $\lambda_{p}$ is the pump wavelength, and $\mathrm{R}(\lambda)$ for $\lambda=\lambda_{s}$ or $\lambda=\lambda_{p}$ is the ratio of cross-sections for emission or absorption. 
The on-off gain of a distributed Raman amplifier DRA, called the overall distributed gain is given by [13]:

$$
G_{d}=\exp \left(g P_{\text {pin }} L_{\text {eff }}\right)
$$

with $L_{\text {eff }}$ the effective fiber length, and is given by [13]:

$$
L_{\text {eff }}=\frac{1-\exp \left(-\alpha_{p} L\right)}{\alpha_{p}}
$$

where $g$ is the Raman gain coefficient, $\alpha_{p}$ is the loss coefficient at the pump wavelength, and $P_{\text {pin }}$ is the pump power.

The noise figure $N F_{D R A}$ for DRA with on-off gain can be written as [13]:

$$
N F_{D R A}=\frac{P_{A S E}}{h \vee \Delta v G_{d}}+\frac{1}{G_{d}}
$$

where $h$ is the Planck's constant, $\Delta v$ is the noise bandwidth, and $P_{A S E}$ is the amplified spontaneous emission ASE power at the fiber output.

The multiple channel amplification, 16 channels in the C-band with a wavelength spacing of $1 \mathrm{~nm}$ were launched to the WDM and a 100-km-long optical fiber. The optical fiber parameters were: effective area $80 \mathrm{um}^{2}$, nonlinear refractive index coefficient $2.6 \times 10^{-20} \mathrm{~m}^{2} / \mathrm{W}$, differential group delay $0.2 \mathrm{ps} / \mathrm{nm} / \mathrm{k}$, dispersion $16.75 \mathrm{ps} / \mathrm{nm} / \mathrm{km}$, and attenuation $0.2 \mathrm{~dB} / \mathrm{km}$. The input power of the signal wavelength was $1 \mathrm{~mW}$.

The layout of the EDFA/DRA hybrid amplifier is shown in Fig. 1. The DRA is composed of an average power amplifier APA in order to decrease computational time by assuming the Raman amplifier that takes into account spontaneous Raman emission noise, Rayleigh back scattering, fiber loss and fiber nonlinearities. Optisystem 7.0 takes into account the forward and backward power of the amplified spontaneous emission ASE according to both the pump power and wavelength. This will allow the calculations of the gain [Eq. (5)] at small segments of fiber length for each input power of the signals. After obtaining the optimum gain, the $N F_{D R A}$ will be found from solving Eq. (7).

The pump laser diode LD was of the $1433.8 \mathrm{~nm}$ wavelength. The right choice of the pump wavelength allows amplifying the WDM signals in their extended aggregate gain spectrum, and then by using the proper choice of a gain equalizer, the lowest noise figure can be obtained.

The second amplifier is the EDFA with the same pump LD wavelength and power. EDFA has two amplification gain blocks separated by an inter-stage gain equalizer (trapezoidal filter) for gain flattening and optical isolators. $\mathrm{NF}$ will be estimated after the gain calculations of the EDFA [Eq. (1)] are done, taking into account all the factors given in the equation and the required optimum values of the fiber in the simulation. The amplified signals by the narrowband hybrid amplifier from the C-band WDM are then ready for the next transmission fiber.

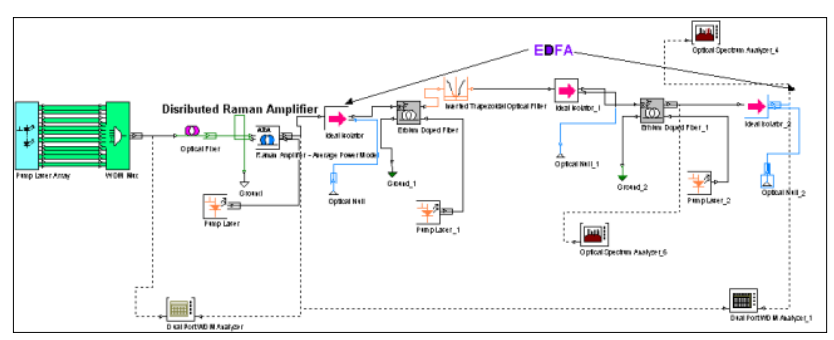

Fig. 1. Optical communication network of $100 \mathrm{~km}$ fiber length and with a hybrid EDFA/DRA optical amplifier.

Before performing the simulation on the network, we have measured the Raman gain coefficient from 0 to $40 \mathrm{THz}$ for the parameters which we have assumed for our calculations in this study. Raman gain is shown in Fig. 2. This gain mechanism in Raman amplification shows how the gain can grow rapidly as a result of pump energy when converted to the fiber. This mechanism can occur in any fiber, and at any signal wavelength but with a properly chosen pump power and wavelength.

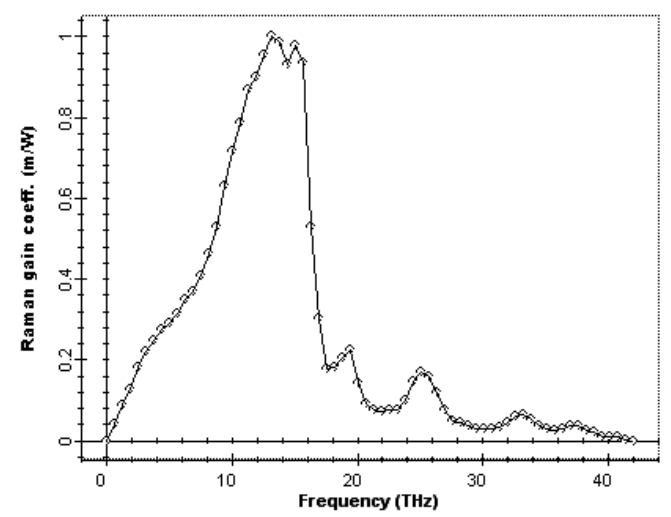

Fig. 2. Raman gain coefficient against the frequency of the fiber used in the simulation.

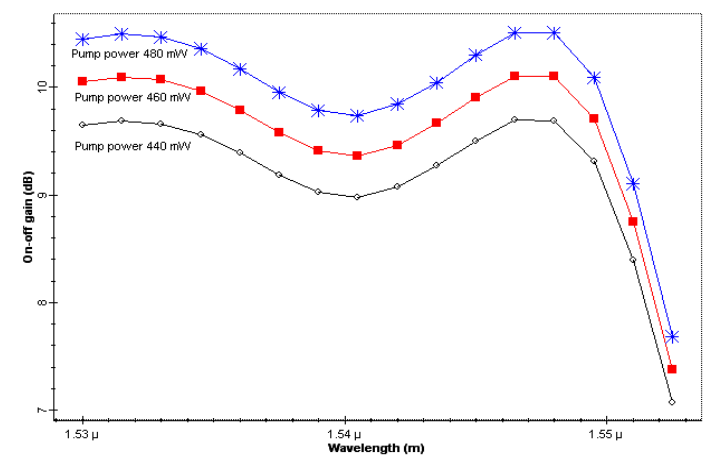

Fig. 3. On-off gain of the hybrid amplifier as a function of wavelength in the C-band, the pump laser power was varied as $440 \mathrm{~mW} ; 460 \mathrm{~mW}$; and $480 \mathrm{~mW}$, while keeping the wavelength constant at $1433.8 \mathrm{~nm}$. 
The optimum pump power and wavelength were varied until the corresponding on-off gains of few $\mathrm{dB}$ (more than $8 \mathrm{~dB}$ ) were achieved for each signal wavelength. The gain flattening of the Raman amplifier itself is of less importance in the hybrid amplifier because the quantity is small compared to that of EDFA and is compensated by the inter-stage filter. This is clear from Fig. 3, where the on-off gain for the C-band was plotted as a wavelength.

The optical signal-to-noise ratio OSNR as a function of signal wavelength is illustrated in Fig. 4. The figure illustrates EDFA/DRA hybrid amplifier (panel A), and EDFA without DRA (panel B), providing that different power of the laser pump at wavelength $1433.8 \mathrm{~nm}$ were launched. As can be seen form the figure, a high OSNR for EDFA/DRA was obtained for the signals that had been increased when the pump power was of higher value.
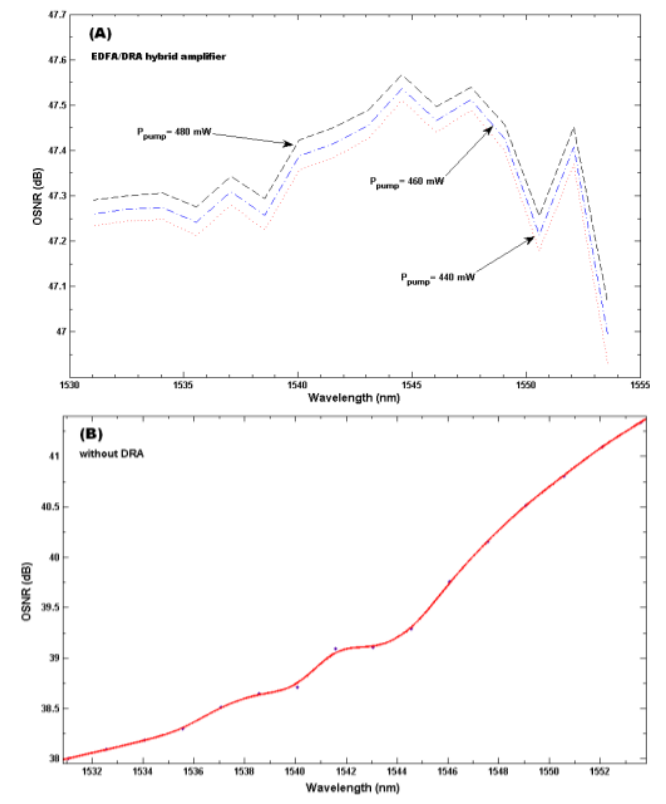

Fig. 4. Optical signal-to-ratio of the hybrid C-band optical amplifier (A) for various pump power of $440 \mathrm{~mW} ; 460 \mathrm{~mW}$; and $480 \mathrm{~mW}$, and without DRA (B).

The noise figure NF of the network was estimated and it is represented in Fig. (5), with the pump power of the Raman, EDFA of $480 \mathrm{~mW}$ and the wavelength constant at $1433.8 \mathrm{~nm}$. The overall NF is below $10 \mathrm{~dB}$ at a wavelength above $1540 \mathrm{~nm}$. This low NF is a limiting case for maximizing the span length and/or minimizing the impairments of fiber nonlinearities. This NF was achieved with a hybrid amplifier for a fiber length of $100 \mathrm{~km}$. The noise figure as low as $4 \mathrm{~dB}$ was attained. The NF is essentially the inverse of the gain spectrum. The overall $\mathrm{NF}$ of the proposed narrowband hybrid amplifier is wavelength dependent. Optimization of NF can be achieved by controlling the fiber length and pump power for a given input power and wavelength.

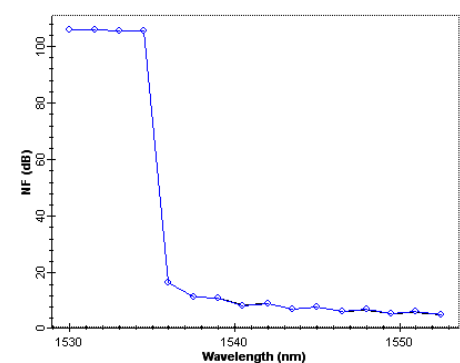

Fig. 5. Overall noise figure NF in the output of the layout used in this study.

It has been reported recently that using some definite system configurations would show a practical implementation and typical positions of the EDFA and DRA in order to enhance the transmission performance [14]. Also, an ultra wideband hybrid optical amplifier utilizing DRA and $\mathrm{C}$ - and extended L-band EDFA amplifiers were shown to realize the $10.8 \mathrm{THz}$ total signal bandwidth [15]. Our simulation of a low NF hybrid amplifier in the C-band proposed and studied numerically. Both DRA and EDFA were pumped by the same LD of wavelength $1433.8 \mathrm{~nm}$. The overall NF of the amplifier was well below 10dB. This NF can be decreased further if extra components are added to the layout. The transmission fiber of our prototype was $100 \mathrm{~km}$ long, connected with 16 channels for this span.

\section{References}

[1] S.K. Kim, S.H. Chang, J.S. Han, M.J. Chu, Electron. Telecom. Res. Inst. J. 24, 81 (2002).

[2] T.N. Nielson, 12th LEOS Annual Meeting, vol. 2, 471 (1999).

[3] T. Suzuki, M. Kakui, M. Hirano, M. Onishi, Y. Nakai, M. Nishimura, Optical Fiber Comm. Conf. MA3 (2001).

[4] S. Kawai, H. Masuda, K. Suzuki, K. Aida, IEEE Photon. Technol. Lett. 11, 886 (1999).

[5] W.Y. Oh, S.S. Lee, H. Lee, W. Seo, European Conf. on Comm. 4.4.5, (2000).

[6] H. Suzuki, J. Kani, H. Masuda, N. Takachio, K. Iwatsuki, Y. Tada, M. Sumida, IEEE Photon. Technol. Lett. 12, 903(2000).

[7] Y. Zhu, W.S. Lee, C. Scahill, C. Fludger, D. Wetly, M. Jones, J. Homan, B. Shaw, A. Hadjifotiou, Electron. Lett. 33, 43(2001).

[8] B. Zhu, P.B. Hansen, L. Leng, S. Stulz, T.N. Nielsen, C. Doerr, A.J. Stentz, D.S. Vengsarkar, Z.J. Chen, D.W. Peckham, L. Gruner-Nielsen, Electron. Lett. 36, 1860 (2000).

[9] H. Masuda, S. Kawai, K.-I Suzuki, Electron. Lett. 35, 411 (1999).

[10] D.A. Chestnut, C. J. S. de Matos, J.R. Taylor, J. Opt. Soc. Am. B- 19, 1901 (2002).

[11] A. Carena, V. Curri, P. Poggiolini, IEEE Photon. Technol. Lett. 13, 1170 (2001).

[12] G.E. Tudury, J. Hu, B.S. Marks, A.S. Lenihan, C.R. Menuk, G.M. Carter, Opt. Comm. 264, 152 (2006).

[13] H. Masuda, Raman Amplifiers for Telecommunications 2, Sub-Systems and Systems (New York, Springer-Verlag 2004).

[14] A.W. Naji, B.A. Hamida, X.S. Cheng, M.A. Mahdi, S. Harun, S. Khan, W.F. AL-Khateeb, A.A. Zaidan, B.B. Zaidan, H. Ahmad, Int. J. Phys.Scien. 6(20), 4674 (2011).

[15] A. Sano, T. Kobayashi, E. Yoshida, Y. Miyamoto, IEICE Trans. Commun. E94-B (2), 400 (2011). 